\title{
Evaluación de la calidad comercial de tres clones de mandioca (Manihot esculenta,crantz) en tres épocas de
}

cosecha.

P. J. Cenóz, G. Martinez y S. Mazza

Facultad de Ciencias Agrarias. U.N.N.E. Sgto. Cabral 2131, (3400) Corrientes. Argentina

\begin{abstract}
Resumen
La calidad comercial de las raíces de mandioca, tiene como indicadores los contenidos de: ácido cianhídrico, humedad, materia seca, almidón, fibra, tiempo de deterioro poscosecha y comportamiento en la cocción. En el Nordeste Argentino la cosecha se efectúa desde marzo hasta agosto, sin embargo la calidad de las raices no es la misma al principio, que al final de dicho periodo. El objetivo del presente trabajo fue evaluar diferentes componentes de calidad en tres épocas de cosecha para tres clones comerciales de la zona. Se probaron los clones: Cambi, Palomita y Catiguá, cosechados en: abril, mayo y junio, en un diseño totalmente al azar con 5 repeticiones. Se analizaron: almidón, ácido cianhídrico, fibra, humedad, materia seca y deterioro poscosecha, realizándose Análisis de Variancia y "test" de Tukey en cada cosecha y el método de Kruskal-Wallis para el deterioro, a cuatro y siete dias de la cosecha Cambi presentó mayor porcentaje de ácido cianhídrico y fibra y menor porcentaje de almidón y materia seca. Palomita presentó menor porcentaje de materia seca que Catiguá, aunque no se diferenciaron en el porcentaje de almidón y ácido cianhídrico. A través de las cosechas: Cambí no modificó su porcentaje de almidón ni ácido cianhídrico, y aumentó el porciento de materia seca. Palomita no modificó el porcentaje de ácido cianhídrico, e incrementó el porciento de almidón y materia seca. Catiguá aumentó el ácido cianhídrico y materia seca y disminuyó almidón. Los clones Cambi y Catiguá presentaron caracteristicas de calidad mas destacados pero en cosecha intermedia (Mayo), en tanto Palomita se comportó mejor en cosecha temprana (Abril ). El deterioro aumentó con los dias poscosecha, presentando Catiguá en los momentos indicados el mayor deterioro y Palomita el menor.
\end{abstract}

Palabras claves : Comercialización, Cosecha, Producción industrial, Mandioca.

\section{Summary}

One of the most important factors in marketing of cassava is root commercial quality; the better indicators are contents of cianhidric acid, humidity, dry matter, fecula, fibre, post harvest deterioration time and cooking behavoir. Harvest is done between March and August in Argentine's N.E.. The objetive of this work was evaluete the different quality component in three harvest time for three commercial clons frequently used in that zone. The clones tested were: Cambi, Palomita y Catiguá, harvested in April, May and June using e completely random design with five replications, determining fecula, cianhidric acid, fibre, humidity, dry matter and post harvest deterioration. Were analized by variance and Tukey test $(=0,05)$, and the post harvest deterioration by Kruskal-Wallis test. at four and seven days for harvest. Cambi has the highest percentages of cianhidric acid and fibre the lowest percentage of dry matter. Palomita, has less dry matter than Catigua but it has not differences in fecula and cianhidric acid percentages . During the harvest time Cambi do not modify neither fecula or cianhidric acid but increases dry matter, Palomita do not modify cianhidric acid but increasses fecula and dry matter. Catigua, ancreasses cianhidric acid and dry matter bur decreasses fecula. Deterioration increasses with post harvest time, being in both moment the biggest in Catigua and the smallest in Palomita.

Keybord: Commercial Harvest, Industrial Producction, Cassava.

\section{Introducción}

La mandioca (Manihot esculenta, Crantz), especie tradicional cultivada en la región del Nordeste argentino para su consumo como fuente de carbohidratos, se cosecha desde el mes de abril hasta fines del invierno. Este período puede durar unos cinco meses, dependiendo de las necesidades del mercado, sin embargo las raíces ya se encuentran en condiciones de cosecha desde el mes de abril. No obstante su calidad comercial no es la misma al principio que al final de dicho periodo, notándose mayor dureza en la estructura de las raíces y una disminución en la calidad culinaria

Según Wheatley (1991) existen varios determinantes de la calidad, tales como contenido de: ácido cianhidrico, materia seca, almidón y fibras, no existiendo estándares de calidad para estas variables.

El mismo autor menciona que el almidón está relacionado con las caracteristicas de textura, consistencia y cocción, aunque no es el simple contenido de almidón el que determina estas caracteristicas sino sus propiedades quimicas (viscosidad, gelatinización etc.) y físicas. El efecto de la edad de la planta sobre la calidad también ha sido evaluada suponiéndose que a mayor edad ocurren cambios fisiológicos que la afectan Wheatley y Gómez (1985) encontraron que el tiempo de cocción aumenta y la calidad culinaria disminuye a medida que la planta es más vieja, a pesar de que sus contenidos de almidón se mantienen estables.

El deterioro poscosecha es un factor importante a tener en cuenta al evaluar la calidad, fundamentalmente por el tiempo demandado para su comercialización. Booth (1974 y 1976) ha estudiado con detenimiento los diferentes pasos del proceso de deterioro, encontrando que el tiempo que transcurre desde la cosecha hasta el inicio dei deterioro está directamente relacionado con factores ambientales: humedad relativa ambiente y temperatura y con la presencia de heridas en las raices.

Los valores promedios encontrados en los distintos clones cultivados en el mundo oscilan entre los siguientes valores: para almidón de 20 a $30 \%$; fibra de 1 a $2 \%$; ácido cianhídrico 100 a 1.000 p.p.m. y materia seca de 35 a $45 \%$. [Montaldo, 1972)

Fue objetivo del presente trabajo evaluar diferentes componentes de calidad en tres épocas de cosecha para tres clones comerciales del Nordeste argentino.

\section{Materiales y Métodos}

La experiencia se realizó en el Campo Experimental de la Facultad de Ciencias Agrarias de la U.N.N.E., ubicado sobre 
la ruta nacional № 12 , a $27^{\circ} 27^{\prime}$ de latitud sur y $58^{\circ} 47^{\prime}$ de longitud oeste; en un clima subhúmedo, mesotermal, con precipitaciones promedios de 1200 a $1300 \mathrm{~mm}$. anuales. El suelo está identificado como Udipsammentes álfico, mixtos, hipertérmicos, pertenecientes a la serie Ensenada Grande (Escobar 1994).

Se probaron los clones de mandioca (Manihot esculenta Crantz): Cambí, Palomita y Catiguá, nombres comunes conocidos en la zona y cultivados por los productores del NE argentino con rendimientos oscilantes entre 10 y 20 tm.ha conforme a la tecnología aplicada.Se utilizó un diseño completamente al azar con cinco repeticiones, y se cosechó a intervalos de treinta dias en los meses de abril, mayo y junio, en las campañas 1994/95 y 1995/96.

La plantación se efectuó en el mes de Setiembre de cada año, a una densidad de 10.000 pl.ha' ${ }^{\prime}$, en parcelas de 40 $\mathrm{m}^{2}$. Las labores de desmalezado se efectuaron en tres oportunidades en forma manual, al igual que la cosecha en los momentos indicados.Los análisis químicos se realizaron conforme a la siguiente metodología: almidón por Krochmal y Kilbrides; ácido cianhídrico por Freymuth (Herce 1954); fibra por Weende (Becker 1961); humedadmateria seca por secado a $100^{\circ} \mathrm{C}$ hasta peso constante y deterioro poscosecha por el método de evaluación propuesto por Marriot et al. (1978).

El método estadístico utilizado para la evaluación de los resultados de los análisis químicos fue análisis de la variancia y "test" de Tukey para las diferentes cosechas y clones.El deterioro poscosecha, es decir la descomposición fisiológica y patológica que sufren las raíces después de cosechada por efecto de la oxigenación. se evaluó mediante el método de Kruskal-Wallis ( Steel, 1992); para lo cual se confeccionó una escala de rangos expresada en la tabla 1. Las evaluaciones se realizaron a los 4 y 7 dias después de cosechadas las raices.

Tabla 1: Escala de rangos para la determinación del deterioro poscosecha.

\begin{tabular}{|c|c|}
\hline DETERIORO (\%) & VALOR ASIGNADO \\
\hline $0,00 \mathrm{a}$ & 0 \\
\hline 10,00 a 29,99 & 1 \\
\hline 30,00 a 59,99 & 2 \\
\hline 60,00 a 79,99 & 3 \\
\hline 80,00 a 89,99 & 4 \\
\hline 90,00 a 100,00 & 5 \\
\hline
\end{tabular}

\section{Resultados y Discusión}

En el Tabla 2 se presentan los promedios de las dos campañas y de las cinco repeticiones de cada clon en cada una de las épocas de cosecha, para los cuatro componentes de calidad estudiados y los resultados del análisis estadístico comparativo de los diferentes clones en cada uno de los momentos de cosecha.

Tabla 2: Resultado del análisis estadístico para los diferentes clones en cada una de las cosechas. (Promedio de dos campañas)

\begin{tabular}{|c|c|c|c|c|c|}
\hline Cosecha & Clon & Almidón(\%) & $\begin{array}{c}\text { Materia seca } \\
(\%)\end{array}$ & $\begin{array}{c}\text { HCN } \\
(\mathrm{mg} / 100 \mathrm{~g})\end{array}$ & Fibra(\%) \\
\hline \multirow{2}{*}{ Abril } & Cambi & $23,32 \mathrm{~b}$ & $37,66 \mathrm{~b}$ & $53,86 \mathrm{a}$ & $2,00 \mathrm{a}$ \\
& Palomita & $28,44 \mathrm{a}$ & $44,96 \mathrm{a}$ & $15,60 \mathrm{~b}$ & $1,43 \mathrm{~b}$ \\
& Catigúa & $27,72 \mathrm{a}$ & $44,04 \mathrm{a}$ & $13,04 \mathrm{a}$ & $1,32 \mathrm{~b}$ \\
\hline \multirow{3}{*}{ Mayo } & Cambí & $27,44 \mathrm{~b}$ & $47,00 \mathrm{a}$ & $49,94 \mathrm{a}$ & $1,08 \mathrm{ab}$ \\
& Palomita & $26,20 \mathrm{~b}$ & $42,76 \mathrm{~b}$ & $15,60 \mathrm{~b}$ & $0,87 \mathrm{~b}$ \\
& Catigúa & $29,24 \mathrm{a}$ & $48,04 \mathrm{a}$ & $13,52 \mathrm{~b}$ & $1,45 \mathrm{a}$ \\
\hline \multirow{3}{*}{ Junio } & Cambí & $26,20 \mathrm{a}$ & $44,98 \mathrm{a}$ & $41,90 \mathrm{a}$ & $0,97 \mathrm{~b}$ \\
& Palomita & $26,16 \mathrm{a}$ & $42,08 \mathrm{~b}$ & $11,74 \mathrm{~b}$ & $1,79 \mathrm{a}$ \\
& Catigúa & $26,44 \mathrm{a}$ & $43,84 \mathrm{a}$ & $19,50 \mathrm{~b}$ & $1,13 \mathrm{~b}$ \\
\hline
\end{tabular}

Letras diferentes indican diferencias significativas entre los promedios $(\alpha=0,05)$

Las comparaciones del análisis estadístico para los promedios de las diferentes cosechas, en cada uno de los clones, se dan en el Tabla 3. 
Tabla 3: Resultados del análisis estadistico para las diferentes cosechas en cada clón. (Promedio de dos campañas)

\begin{tabular}{|c|c|c|c|c|c|}
\hline Cosecha & Clon & Almidón(\%) & $\begin{array}{c}\text { Materia seca } \\
(\%)\end{array}$ & $\begin{array}{c}\text { HCN } \\
(\mathrm{mg} / 100 \mathrm{~g})\end{array}$ & Fibra(\%) \\
\hline \multirow{3}{*}{ Cambi } & Abril & $23,32 \mathrm{~b}$ & $37,66 \mathrm{a}$ & $53,86 \mathrm{a}$ & $2,00 \mathrm{a}$ \\
& Mayo & $27,44 \mathrm{a}$ & $47,00 \mathrm{~b}$ & $49,94 \mathrm{a}$ & $1,08 \mathrm{~b}$ \\
& Junio & $26,20 \mathrm{a}$ & $44,98 \mathrm{ab}$ & $41,90 \mathrm{~b}$ & $0,97 \mathrm{~b}$ \\
\hline \multirow{3}{*}{ Palomita } & Abril & $28,44 \mathrm{a}$ & $44,96 \mathrm{a}$ & $15,60 \mathrm{a}$ & $1,43 \mathrm{ab}$ \\
& Mayo & $26,20 \mathrm{~b}$ & $42,76 \mathrm{~b}$ & $15,60 \mathrm{a}$ & $0,87 \mathrm{a}$ \\
& Junio & $26,16 \mathrm{~b}$ & $42,08 \mathrm{~b}$ & $11,74 \mathrm{~b}$ & $1,79 \mathrm{~b}$ \\
\hline \multirow{3}{*}{ Catiguá } & Abril & $27,72 \mathrm{~b}$ & $44,04 \mathrm{a}$ & $13,00 \mathrm{a}$ & $1,32 \mathrm{a}$ \\
& Mayo & $29,24 \mathrm{~b}$ & $48,04 \mathrm{~b}$ & $13,52 \mathrm{a}$ & $1,45 \mathrm{a}$ \\
& Junio & $20,44 \mathrm{a}$ & $43,84 \mathrm{a}$ & $19,50 \mathrm{~b}$ & $1,13 \mathrm{a}$ \\
\hline
\end{tabular}

Letras diferentes indican diferencias significativas entre los promedios $(\alpha=0,05)$

La Tabla 4 muestra los valores de deterioro encontrados según la escala propuesta (Tabla 1) a los cuatro y siete dias, en promedio para cada clon.

Tabla 4: Valores promedios de deterioro poscosecha (Promedio de dos campañas)

\begin{tabular}{|c|c|c|c|c|}
\cline { 2 - 5 } \multicolumn{1}{c|}{} & \multicolumn{2}{c|}{4 días } & \multicolumn{2}{c|}{7 días } \\
\hline Clón & Deterioro(\%) & Valor & Deterioro(\%) & Valor \\
\hline Cambí & 0,00 & 0 & 40,00 & 2 \\
\hline Palomita & 0,00 & 0 & 30,00 & 2 \\
\hline Catiguá & 15,00 & 1 & 60,00 & 3 \\
\hline
\end{tabular}

De los resultados se deduce que los valores de ácido cianhídrico no se diferenciaron significativamente en un mismo clon en las diferentes cosechas, excepto Catiguá que elevó su contenido en la cosecha de Junio. Entre los diferentes clones se encontraron diferencias significativas, presentando Cambi mayor contenido que los otros en las tres cosechas. Sin embargo, estos valores, se encuentran dentro de los normales para las denominadas mandiocas dulces es decir menos de 500 p.p.m. (Montaldo 1979)

En lo que respecta a almidón, Cambi y Catiguá presentan los mayores valores en la segunda cosecha y Palomita en la primera.

Con respecto al contenido de materia seca, Cambí presentó diferencias entre la primera y la última cosecha, incrementando los valores con el tiempo; en Catiguá, el mayor valor se dió en la segunda cosecha, que sólo se diferenció de la última; para Palomita la primer cosecha superó a las otras dos.

Los valores de fibra estarian indicando para Cambi un menor contenido, por to tanto un mejor momento de cosecha en la segunda y tercera, disminuyendo el mismo con el tiempo; en Catiguá no se encontraron diferencias entre las cosechas; para Palomita el menor valor se dió en la segunda cosecha que se diferenció significativamente de la última.

Para el análisis del deterioro poscosecha se adoptó la escala propuesta en la Tabla 1 , los resultados por clon, evaluados a los 4 y 7 dias después de cosechados figuran en el Tabla 4. Cambi y Palomita no presentaron sintomas de deterioro a los 4 dias, en cambio si lo hizo Catiguá.
Para la evaluación a los 7 dias los porcentajes fueron variables siendo el clon Palomita el menos afectado y Catiguá el de mayor deterioro.

\section{Conclusiones}

Los clones Cambi y Catiguá presentaron caracteristicas de calidad más destacadas para una cosecha intermedia (Mayo), en tanto Palomita se comporta mejor en cosecha temprana (Abril).El deterioro poscosecha a los siete dias fue mayor en Catiguá lo que reduce su tiempo de comercialización. Presentando el mejor comportamiento en este aspecto el clon Palomita.

\section{Bibliografía.}

Becker, M. 1961. Análisis y valoración de piensos y forrajes. Editorial Acribia. España

Booth, R.H. 1974. Post-harvesdeterioration of tropical root crops, losses and their control. Tropical Science 16: 49-63.

Booth, R.H. 1976. Storange of fresh cassava. Postharvest deterioration and its control Experimental Agriculture. 12: 103-111.

Escobar, E.H.; D Ligier; R. Melgar; H. Matteio; O.Vallejos. 1994. Mapa de Suelo de los Departamentos de Capital, San Cosme e Itatí de la Provincia de Corrientes. Publicación del Instituto Nacional de Tecnología Agropecuaeria (I.N.T.A.).

Herce, P. 1954. Análisis Agricola. Editorial Donat. 


\section{España.}

Marriott, J.; B.O. Been and C, Perkins. 1978. The aetiology of vascular discolouration in cassava roots after harvesting association with water loss from wound. Physiologia Plantarum 44: 38-42.

Montaldo, A. 1972 Cultivo de raices y tubérculos tropicales. I.I.C.A. Lima. Perú. 284 pag.

Montaldo, A. 1979. La Yuca o Mandioca Cultivo, industrialización, aspectos económicos, empleo en la alimentación animal. Mejoramiento. I.I.C.A. Instituto Interamericano de Ciencias Agrícolas). Costa Rica. 381 pag.

Steel, R.G.D.; Torrie, J.M.. 1992. Bioestadistica: Principios y procedimientos. Editorial Grawn Hell. Méjico.

Wheatley C.C. 1991. Calidad de raices de yuca y factores que intervienen el ella. Mejoramiento Genético de la Yuca en América Latina. Centro Internacional de Agricultura Tropical (C.I.A.T.) Colombia. pag. 267-291.

Wheatley C.C.; Gómez G.G. 1985. Evaluation of some quality characteristic in cassava storage root. Qualitas Plantarum Plant Food for Human Nutrition 35 : pag. 121129.

Wheatley C.C.; Lozano C.; Gómez, G. 1986. Deterioración poscosecha y almacenamiento de raices de yuca. Yuca: investigación, producción y utilización. Centro Internacional de Agricultura Tropical (C.I.A.T.) Documento de Trabajo NN 50: pag 493-511. 